\title{
M/G/1 Feedback Queue When Server is Off and on Vacation
}

\author{
S.Shanmugasundaram ${ }^{1}$, G.Sivaram² \\ ${ }^{1}$ Assistant Professor, Department of Mathematics, Government Arts College, Salem - 636007 , India. \\ ${ }^{2}$ Associate Professor, Department of Mathematics, Government Arts College, Salem - 636 007, India.
}

\begin{abstract}
In this paper, we study a M/G/1 queue with feedback for a customer and when the server is on Vacation. The steady state probabilities are obtained and other operating characteristics are also Obtained. Finally the numerical example is provided to check the correctness.
\end{abstract}

Keywords: Bernoulli process, feedback, customers,vacation , steady state probability

\section{INTRODUCTION}

The queueing theory or waiting line theory owes its development to A.K.Erlang. The Erlang work [1] on queueing stimulated many authors to develop a variety of queueing models. A queue is a waiting line in which a group of customers /items wait in front of a service channel. The queue does not include a customer being serviced. Many queueing situations have features that the customer may be served once again. The customer may or may not opt for a feedback. When the server is busy, the arriving customers do not line up or leave the system immediately forever and the customers go to some virtual place which is called as orbit and try at a later time. Queueing system with retrial occurs frequently in practice. In the year 1963 Takacs[8] first introduced the concept of feedback mechanism in queues which includes the possibility for a customer to return to the counter for additional service. In queueing system, a vacation is one in which a server may become unavailable for a random period of time from a service channel. Many researchers are also concentrating on queueing models with vacation policies.

In 1996 Gautam Choudhury [2] have proposed a two phase queueing system with Bernoulli feedback.. In the year 1998, Krishna Reddy, Nadarajan and Arumuganathan [4] have investigated an $\mathrm{M}^{*} / \mathrm{G}(\mathrm{a}, \mathrm{b}) / 1$ queueing model with N-policy, general setup times and multiple vacations. In the year 2000 , Santhakumaran and Thangaraj [5] have studied a single server queue with impatient and feedback customers. Thangaraj and Vanitha[10] have focused on a continued fraction approach to a $M / M / 1$ queue with feedback. In the year 2008, Santhakumaran and Shanmugasundaram [6] made a study on a Preparatory work on arrival customers with a single server feedback queue.
Queueing theory analysis involves the study of systems over time. Time dependent analysis helps to understand the behavior of a system when the parameters involved are perturbed and it can contribute to the costs and benefits of operating a system. Parthasarathy [14] and then Parthasarathy and Sharafali [15] have focused to study single and multiple server poisson queues of transient state solution. Krishna Kumar and Arivudainambi [9] have proposed a transient state solution for the mean queue size of $\mathrm{M} / \mathrm{M} / 1$ queueing model when catastrophes occur at the service station.

Shanmugasundaram and Shanmugavadivu [12 ] have made a study on a time dependant solution of single server queue with Markovian arrival and Markovian service. Shanmugasundaram and Chitra [13] have discussed time dependent solution of $\mathrm{M} / \mathrm{G} / 1$ retrial queue and feedback on non retrial customers with catastrophes. Chandrasekaran and Saravanarajan [3] have studied a transient and reliability analysis of a single server queue with feedback subject to catastrophes .Shanmugasundaram and Chitra [11] have studied a time dependent solution of a single server feedback queue with server on vacation. Kaliappan Kalidass and Kasturi Ramnath [7] have discussed transient analysis of an $\mathrm{M} / \mathrm{M} / 1$ queue with multiple vacations.

\section{Description of the model and Analysis}

In this model, we are concerned with the random processes in which the external customers arrive according to a poisson process with rate $\lambda$. The service for an arriving customer begins instantaneously if the server is idle upon an arrival.

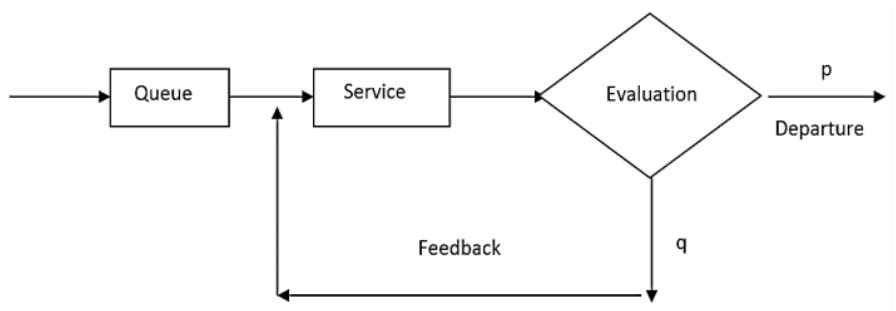

Fig-1

In the above figure, after getting the service, depending on the level of service a decision is made whether to depart or feedback. If a customer does feedback, he joins the feedback stream with probability $q$ and joins the end of the queue. It is 
assumed that feedback occurs instantaneously. If a customer decides not to feedback, he departs with probability $p(p+q=1)$. The capacity of the queue is infinite and the queue discipline is first come first served.

The service times are independent and identically distributed and is given by

$$
\mu= \begin{cases}\mu_{1}, & \text { if the customer is served with feedback. } \\ \mu_{2}, & \text { if the customer is served without feedback. }\end{cases}
$$

The server may be away from the service centre for a vacation and customers who arrive while the server is on vacation will have to wait until he returns from vacation. The server goes for vacation if he completes all the services. The vacation time extends when no other customer joins the queue. The vacation time is an exponentially distributed random variable with rate $\Omega$. The motivation for this model comes from bank, hospital, production system etc.

Let $p_{n}(0)$ and $p_{n}(1)$ denote the respective probabilities that there are ' $n$ ' customers in the system when the server is in vacation and when the server is available. Let $\mathrm{L}_{0}(\mathrm{z})=$ $\sum_{n=1}^{\infty} p_{n}(0) z^{n}$ and $\mathrm{L}_{1}(\mathrm{z})=\sum_{n=1}^{\infty} p_{n}(1) z^{n}$ be the probability generating functions for $|z| \leq 1$

Generally it is assumed that the server is busy with $\mathrm{i}$ customers and $p_{i}(1)=\alpha_{i}$ for $i \geq 1$ and $p_{i}(0)=0$ for $i \geq 1$ where $\alpha_{i}$ is the probability of $i$ customers initially

The system of (differential-difference) equations are

$-\lambda \mathrm{p}_{0}(0)+\left[\mathrm{p} \mu_{1}+\mathrm{q} \mu_{2}\right] \mathrm{p}_{1}(1)=0$

For $\mathrm{n}=1,2,3 \ldots \ldots$

$-(\lambda+\Omega) p_{n}(0)+\lambda p_{n-1}(0)=0$

$-\left[\lambda+p \mu_{1}+q \mu_{2}\right] p_{1}(1)+\Omega p_{1}(0)+\left[p \mu_{1}+q \mu_{2}\right] p_{2}(1)=0$

For $n=2,3,4 \ldots \ldots \ldots$

$-\left[\lambda+p \mu_{1}+q \mu_{2}\right] p_{n}(1)+\Omega p_{n}(0)+\left[p \mu_{1}+q \mu_{2}\right] p_{n+1}(1)+\lambda p_{n-1}(1)=0$

From equation (1), we get

$\mathrm{p}_{1}(1)=\frac{\lambda \mathrm{p}_{0}(0)}{\mathrm{p} \mu 1+\mathrm{q} \mu 2}$,

From equation (2), we get

$(\lambda+\Omega) \mathrm{p}_{\mathrm{n}}(0)=\lambda \mathrm{p}_{\mathrm{n}-1}(0)$

$\mathrm{p}_{\mathrm{n}}(0)=\frac{\lambda}{\lambda+\Omega} \mathrm{p}_{\mathrm{n}-1}(0)$

Recursively using this equation, we get

$\mathrm{p}_{\mathrm{n}}(0)=\left(\frac{\lambda}{\lambda+\Omega}\right)^{n} \mathrm{p}_{0}(0)$
From equation (1), we get

$p_{n+1}(1)=\frac{\left(\lambda+p \mu_{1}+q \mu_{2}\right)}{p \mu_{1}+q \mu_{2}} p_{n}(1)-\frac{\lambda p_{n-1}(1)}{p \mu_{1}+q \mu_{2}}-\frac{\Omega p_{n}(0)}{p \mu_{1}+q \mu_{2}}$

The probability generating function $\mathrm{L}_{1}(\mathrm{z})$ is

$\left[\lambda z+\frac{p \mu_{1}+q \mu_{2}}{z}-\left(\lambda+p \mu_{1}+q \mu_{2}\right) z\right] Q 1(z)+\Omega L_{0}(z)-\left(p \mu_{1}+q \mu_{2}\right)$

$\mathrm{p}_{1}(1)-\Omega \mathrm{p}_{0}(0)=0$

$\frac{Q_{1(z)}}{z}\left[\lambda z^{2}+\left(p \mu_{1}+q \mu_{2}\right)-\left(\lambda+p \mu_{1}+q \mu_{2}\right) z\right]$

$=\left(p \mu_{1}+q \mu_{2}\right) p_{1}(1)+\Omega p_{0}(0)-\Omega L_{0}(z)$

$\mathrm{L}_{1}(\mathrm{z})=\frac{z\left[\left(\mathrm{p} \mu_{1}+\mathrm{q} \mu_{2}\right) \mathrm{p}_{1}(1)+\Omega \mathrm{p}_{0}(0)-\Omega \mathrm{L}_{0}(\mathrm{z})\right]}{\lambda \mathrm{z}^{2}-\left(\lambda+\mathrm{p} \mu_{1}+\mathrm{q} \mu_{2}\right) \mathrm{z}+\mathrm{p} \mu_{1}+\mathrm{q \mu} \mu_{2}}$

Equating the denominator to zero

$\lambda z^{2}-\left(\lambda+p \mu_{1}+q \mu_{2}\right) z+p \mu_{1}+q \mu_{2}=0$ whose roots are

$\mathrm{Z}_{1}=\frac{w-\sqrt{w^{2}-4 \lambda\left(\mathrm{p} \mu_{1}+\mathrm{q} \mu_{2}\right)}}{2 \lambda}$ and

$\mathrm{z}_{2}=\frac{w+\sqrt{w^{2}-4 \lambda\left(\mathrm{p} \mu_{1}+\mathrm{q} \mu_{2}\right)}}{2 \lambda} \quad$ where $\mathrm{w}=\lambda+\mathrm{p} \mu_{1}+\mathrm{q} \mu_{2}$

The probabilities for the single server feedback queue are

$$
\begin{aligned}
& \mathrm{P}_{\mathrm{n}}(1)=\frac{\lambda^{n}}{\left(\mathrm{p} \mu_{1}+\mathrm{q} \mu_{2}\right)^{n}}\left[1-\frac{\lambda}{\left(\mathrm{p} \mu_{1}+\mathrm{q} \mu_{2}\right)}\right] \frac{\lambda}{\lambda+\Omega} \\
& \mathrm{P}_{\mathrm{n}}(1)=\rho^{\mathrm{n}}(1-\rho) \frac{\lambda}{\lambda+\Omega} \quad \text { where } \quad \rho=\frac{\lambda}{\mathrm{p} \mu_{1}+\mathrm{q} \mu_{2}}
\end{aligned}
$$

\section{Operating Characteristics}

In this section, we obtain the following by simple straight forward calculations

If $\mathrm{n}$ denotes the number of customers in the system with instantaneous Bernoulli process, the average number of customers in the system is

$\mathrm{E}(\mathrm{n})=\sum_{0}^{\infty} n\left[p_{n}(0)+p_{n}(1)\right]$

$\mathrm{E}(\mathrm{n})=\sum_{0}^{\infty} n\left[\rho^{\mathrm{n}}(1-\rho) \frac{\lambda}{\lambda+\Omega}+\left(\frac{\lambda}{\lambda+\Omega}\right)^{n}(1-\rho)\right]$

$\mathrm{E}(\mathrm{n})=(1-\rho) \frac{\lambda}{\lambda+\Omega} \sum_{0}^{\infty} n \rho^{\mathrm{n}}+(1-\rho) \sum_{0}^{\infty} \mathrm{n}\left(\frac{\lambda}{\lambda+\Omega}\right)^{n}$

By Little's formula, the other characteristics are

$\mathrm{E}(\mathrm{m})=\lambda(1-\rho) \frac{\lambda}{\lambda+\Omega} \sum_{0}^{\infty} n \rho^{\mathrm{n}}+(1-\rho) \lambda \sum_{0}^{\infty} \mathrm{n}\left(\frac{\lambda}{\lambda+\Omega}\right)^{n}-\frac{\lambda}{\mathrm{p} \mu_{1}+\mathrm{q} \mu_{2}}$

$\mathrm{E}(\mathrm{v})=(1-\rho) \frac{1}{\lambda+\Omega} \sum_{0}^{\infty} n \rho^{\mathrm{n}}+\frac{(1-\rho)}{\lambda} \sum_{0}^{\infty} \mathrm{n}\left(\frac{\lambda}{\lambda+\Omega}\right)^{n}$ 


\section{NUMERICAL STUDY}

Based on the mean response time of the model, a numerical study is made. The influence of the parameters $p$ and $q$ on the mean response time is illustrated.

The values of $\mathrm{p}_{\mathrm{n}}(0)$ is computed with the fixed time $\Omega=$ $0.3,0.5,0.7$ with parameters $\mathrm{p}=0.6$ and $\mathrm{q}=0.4$. For this purpose we have taken the values of $\mu_{1}=15$ and $\mu_{2}=15$

Table 1

\begin{tabular}{|l|l|l|l|}
\hline$\lambda$ & $\Omega(0.3)$ & $\Omega(0.5)$ & $\Omega(0.7)$ \\
\hline 2 & 0.753623 & 0.693333 & 0.641975 \\
\hline 3 & 0.727273 & 0.685714 & 0.648649 \\
\hline 4 & 0.682171 & 0.651852 & 0.624113 \\
\hline 5 & 0.628931 & 0.606061 & 0.584795 \\
\hline 6 & 0.571429 & 0.553846 & 0.537313 \\
\hline 7 & 0.511416 & 0.497778 & 0.484848 \\
\hline 8 & 0.449799 & 0.439216 & 0.429119 \\
\hline 9 & 0.387097 & 0.378947 & 0.371134 \\
\hline 10 & 0.323625 & 0.31746 & 0.311526 \\
\hline 11 & 0.259587 & 0.255072 & 0.250712 \\
\hline 12 & 0.195122 & 0.192 & 0.188976 \\
\hline
\end{tabular}

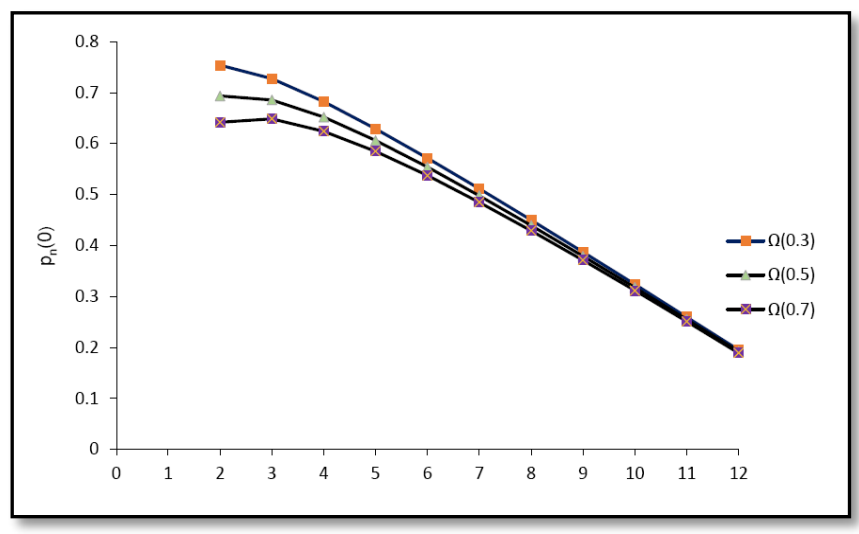

Fig-2

The values of $p_{\mathrm{n}}(1)$ is computed with the fixed time $\Omega=0.3,0.5,0.7$ with parameters $\mathrm{p}=0.6$ and $\mathrm{q}=0.4$. For this purpose we have taken the values of $\mu_{1}=15$ and $\mu_{2}=15$

Table :2

\begin{tabular}{|l|l|l|l|}
\hline$\lambda$ & $\Omega(0.3)$ & $\Omega(0.5)$ & $\Omega(0.7)$ \\
\hline 2 & 0.100483 & 0.092444 & 0.085597 \\
\hline 3 & 0.145455 & 0.137143 & 0.12973 \\
\hline 4 & 0.181912 & 0.173827 & 0.16643 \\
\hline 5 & 0.209644 & 0.20202 & 0.194932 \\
\hline
\end{tabular}

\begin{tabular}{|l|l|l|l|}
\hline$\lambda$ & $\Omega(0.3)$ & $\Omega(0.5)$ & $\Omega(0.7)$ \\
\hline 6 & 0.228571 & 0.221538 & 0.214925 \\
\hline 7 & 0.238661 & 0.232296 & 0.226263 \\
\hline 8 & 0.239893 & 0.234248 & 0.228863 \\
\hline 9 & 0.232258 & 0.227368 & 0.22268 \\
\hline 10 & 0.21575 & 0.21164 & 0.207684 \\
\hline 11 & 0.190364 & 0.187053 & 0.183856 \\
\hline 12 & 0.156098 & 0.1536 & 0.151181 \\
\hline
\end{tabular}

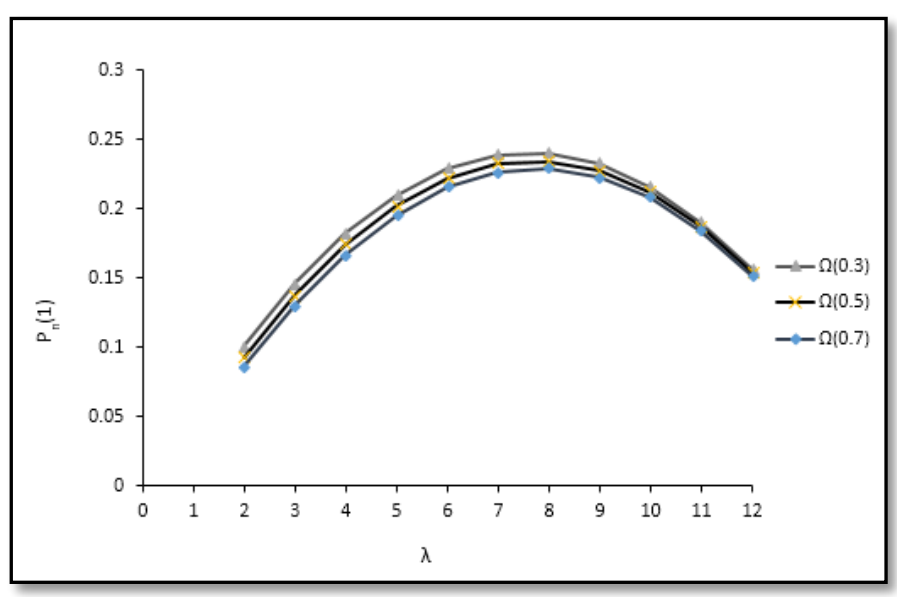

Fig-3

\section{CONCLUSION}

We have derived the probability generating function, the steady state probabilities and the operating characteristics. The numerical examples illustrate that the arrival rate increases $\mathrm{p}_{\mathrm{n}}(0)$, fig-2( probability of $\mathrm{n}$ customers in the system when the server is on vacation) decreases and $\mathrm{p}_{\mathrm{n}}(1)$ ,fig-3(probability of $n$ customers in the system when the server is available)changes. This shows the feasibility of the model.

\section{REFERENCES}

[1] A.K.Erlang, The Theory of probabilities and Telephone conversations, Nyt Jindsskriff for Mathematic, B 20, 33-39 (1909).

[2] Gautam Choudhury and MadhuChanda Paul, A Two phase queueing system with Bernoulli feedback, International Journal of Information and Management Sciences, Vol . 16, pp.35-52, 2005.

[3] V.M. Chandrasekaran, M.C.Saravanarajan, Transient and Reliability analysis of $\mathrm{M} / \mathrm{M} / 1$ feedback queue subject to catastrophes, server failures and repairs, Volume 77 No.5(2012) pp: 605-622.

[4] G.V.KrishnaReddy, R.Nadarajan and R.Arumuganathan, Analysis of Bulk queue with N policy multiple vacations and setup time, Computer 
Operations Research, Vol. 25, pp.954-967, 1998.

[5] A. Santhakumaran, and V.Thangaraj., A single server queue with impatient and feedback customers, International Journal of Information and Management Sciences, Vol. 11, pp.71-79,2000.

[6] A.Santhakumaran, and S.Shanmugasundaram, Preparatory Work on Arrival Customers with a Single Server Feedback Queue. Journal of Information and Management Sciences Vol. 19, No.2, pp.301-313, June, 2008.

[7] Kaliappan Kalidass and Kasturi Ramanath, Transient Analysis of an M/M/1 Queue with Multiple Vacations,Pak.j.stat.oper.res. Vol. X No.1(2014), pp: 121-130.

[8] L.Takacs, A single Server Queue with feedback, The Bell System Technical Journal, Vol. 42,pp. 505-519, 1963.

[9] B.Krishna Kumar,D.Arivudainambi, Transient Solution of an $\mathrm{M} / \mathrm{M} / 1$ Queue with catastrophes, Computer and Mathematics with Applications, 40(2000), pp: 1233-1240.

[10] V.Thangraj, S.Vanitha, M/M/1 queue with feedback a continued fraction approach, International Journal of Computational and Applied Mathematics, 5(2010), 129-139.

[11] S.Shanmugasundaram, S.Chitra, Time dependent solution of a single server feedback queue with server is on vacation,International Journal for Research n Mathematics and Statistics, Vol.2,March 2016.

[12] S.Shanmugasundaram and A. Shanmugavadivu, A Study on Time Dependent Solution of Single Server Queue with Markovian Arrival and Markovian Service, CiiT International Journal of Data Mining and Knowledge Engineering, Vol3, No.16(2011),pp: 981-984.

[13] S.Shanmugasundaram, S.Chitra, Time dependent solution of $\mathrm{M} / \mathrm{G} / 1$ retrial queue and feedback on Non Retrial customers with catastrophes, Global Journal of Pure and Applied Mathematics, Vol 11,No.1(2015)pp: 90-95.

[14] P.R. Parthasarathy, A transient solution to a $M / M / 1$ queue, A simple Approach,(Adv.Appl.Prob.19(1987) pp: 997-998.

[15] P.R. Parthasarathy and M.Sharafali, Transient solution to the many server Poisson queues, J.Appl.Prob. 26,(1989), pp: 584-594. 